\title{
Tripiana-Muñoz, S. (2019). Estrategias eficaces de práctica instrumental. Primeros pasos al estudiar una obra musical. Granada: Editorial Libargo
}

Tras largas discusiones a lo largo del tiempo entre las diversas claves del éxito de los grandes intérpretes, esta monografía aborda el tema de la práctica instrumental desde la perspectiva de la importancia del estudio eficaz, estratégico y perseverante, y, de este modo, la perentoria necesidad de convertirse en objeto de investigación. La autora relata cómo, en tan sólo los últimos cien años, se ha observado un interés científico por la naturaleza de la práctica instrumental y su relación con la transición desde niveles iniciales hasta niveles de excelencia, por medio de un cuerpo creciente de estudios a través de numerosas disciplinas (Barry y Hallam, 2002). No obstante, señala que, según la revisión de Gabrielsson (2003), los estudios sobre la planificación de la interpretación, es decir, sobre cómo formar representaciones mentales de la música, diseñar planes interpretativos y estrategias adecuadas de práctica instrumental, no comenzaron hasta después de la aparición de la psicología cognitiva en la segunda mitad del siglo $\mathrm{XX}$, ya que había poco lugar para tales cuestiones en la corriente conductista.

Desde 1975, el número de estudios publicados sobre estrategias de práctica instrumental ha ido aumentando cada década y, según Jorgensen (2004), aproximadamente dos tercios de los trabajos publicados desde 1916 hasta nuestros días datan de 1990 o son, incluso, más tardíos. En los últimos veinte años, la investigación acerca de la práctica instrumental se ha fundamentado en las tendencias descritas en la psicología social y cognitiva (Santos y Hentschke, 2009). Estos estudios demuestran el potencial de planificación intencional de los músicos para hacer frente a las situaciones de la práctica y, a su vez, ayudan a sistematizar aspectos que permitan la mejora y el perfeccionamiento de su nivel de experiencia músico-instrumental.

El libro consta de los siguientes capítulos:

Capítulo 1. Buscando estrategias de práctica instrumental. La autora expone su relación personal con el tema objeto de estudio y justifica convenientemente el porqué de su investigación. Asimismo, de modo ameno y didáctico conduce su exposición dando respuesta a algunos grandes interrogantes que sobrevuelan el pensamiento de aquellas personas dedicadas a la interpretación musical: ¿se nace con talento musical o es el músico el único responsable de su propio éxito? Cualquier gran instrumentista ¿ha logrado alcanzar su nivel de dominio técnicointerpretativo con menor dedicación que el resto de los mortales? ¿existen diferencias de género a la hora de llevar a cabo la práctica instrumental? ¿para qué practica un pianista, una percusionista o una flautista? ¿cuál es el propósito principal de las horas de estudio?

De esta manera, desde el capítulo 2 hasta el 9, se presentan las ocho estrategias de práctica que guiarán el estudio de una nueva obra musical. Tras su documentada definición, la autora revela diversas investigaciones previas al respecto de cada una de las estrategias; relata cómo

@MARTA VELA. THE CONTENT OF THIS ARTICLE IS THE SOLE RESPONSIBILITY OF THE AUTHORS. THE REVISTA ELECTRÓNICA DE LEEME AND UNIVERSITAT DE VALĖNCIA ARE NOT LIABLE FOR ANY LEGAL ACTIONS THAT MAY ARISE INVOLVING THE ARTICLE'S CONTENT. REVISTA ELECTRÓNICA DE LEEME - LSTA ELECTRÓNICA EUROPEA DE MÚSICA EN LA EDUCACIÓN.HTTP://OJS.UV.ES/INDEX/PHP/LEEME/INDEX. ISSN: 1575-9563. EDITORES: UNIVERSIDAD DE VALENCIA Y JESÚS TEJADA GIMÉNEZ. VISIBILIDAD DE ESTA REVISTA: SCOPUS, EMERGING SOURCES CITATION INDEX (CLARIVATE), EBSCO, CINDOC (CSIC), CITEFACTOR, COPAC, DIALNET, DICE (CSIC), DOAJ, E-REVISTAS (CSIC), EBSCO PREMIER, ERIH+, GALE CENGAGE LEARNING, IN-RECS, IRESIE, LATINDEX, MIAR, OCLC WORLDCAT, RESH, REDIB, RILM CORE JOURNALS, SUDOC, ULRICHS, ESTA REVISTA ESTÁ PUBLICADA CON EL APOYO INSTITUCIONAL DE REDIRIS-CONSEJO SUPERIOR DE INVESTIGACIONES CIENTIFICAS Y ES DE ACCESO LIBRE. CREATIVE COMMONS LICENSE 4.0 BY 


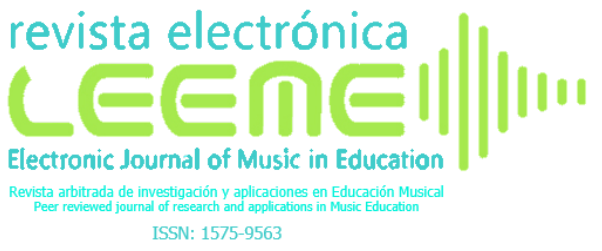

SECCIÓN DIFUSIÓN DE PUBLICACIONES: MARTA VELA

llevar a cabo cada estrategia por medio de diferentes propuestas; expone importantes testimonios de uso de cada estrategia por grandes intérpretes de música; y, finalmente, aborda un práctico resumen de cada una de ellas para llevar a la práctica y optimizar el estudio musical más eficazmente.

Capítulo 2. Práctica fragmentada: la estrategia más sencilla. Esta primera propuesta propone una ejecución fragmentada, según criterios musicales, dividiendo la complejidad y alentando a progresar al intérprete desde lo más simple a lo más complejo.

Capítulo 3. Práctica con máxima concentración: la estrategia más agotadora. En esta propuesta la autora pone de relieve la importancia de la atención plena en la ejecución musical.

Capítulo 4. Adecuación ergonómica: la estrategia más natural. Un interesante relato en el que se pone de manifiesto el cuerpo humano y el uso que se hace del mismo como un ingrediente más de la práctica musical exitosa. En definitiva, hace alusión al funcionamiento cómodo y natural del medio corporal de cualquier instrumentista por medio del movimiento natural, la economía motriz (máximo rendimiento y mínimo esfuerzo), la influencia de la gravedad, integrando la respiración y buscando el apoyo como medio de seguridad.

Capítulo 5. Mensajes de auto-orientación: la estrategia más constructiva. En este capítulo, la autora hace un recorrido por el contenido de mensajes de auto ayuda que plantean objetivos durante el estudio, que sirven de auto-guía como mensajes de advertencia, de apoyo o de recuerdo y que efectúan una retroalimentación informativa evaluando los resultados o la misma sesión de práctica.

Capítulo 6. Escucha autocrítica: la estrategia más analítica. Con esta propuesta la autora pone de relieve la importancia de percibir auditivamente cómo cada sonido resuena en el espacio exterior, efectuando una necesaria retroalimentación y pudiendo valerse de la ayuda de medios audiovisuales, principalmente grabaciones de audio y vídeo.

Capítulo 7. Lectura precisa: la estrategia más preventiva. En este caso, propone realizar una lectura precisa de la obra musical objeto de estudio, a modo preventivo, tocando con corrección desde la primera vez, aislando la dificultad, analizando su naturaleza y asumiendo un margen de aceptabilidad sin que la perfección se convierta en obsesión para el intérprete.

Capítulo 8. Práctica indirecta: la estrategia más extendida. Esta repetida propuesta supone alterar el tempo de la interpretación, generando nuevos retos a resolver por la persona que interpreta y acrecentando la dificultad existente en la partitura.

Capítulo 9. Respeto al límite actual: la estrategia más prudente. En este capítulo la autora pretende que cualquier instrumentista efectúe una adaptación a su límite técnico-interpretativo actual con el instrumento, es decir, que no pretenda más de lo que en el momento presente es capaz de realizar.

@MARTA VELA. THE CONTENT OF THIS ARTICLE IS THE SOLE RESPONSIBILITY OF THE AUTHORS. THE REVISTA ELECTRÓNICA DE LEEME AND UNIVERSITAT DE VALĖNCIA ARE NOT LIABLE FOR ANY LEGAL ACTIONS THAT MAY ARISE INVOLVING THE ARTICLE'S CONTENT. REVISTA ELECTRÓNICA DE LEEME - LSTA ELECTRÓNICA EUROPEA DE MÚSICA EN LA EDUCACIÓNHTTP://OJS.UV.ES/INDEX/PHP/LEEME/INDEX. ISSN: 1575-9563. EDITORES: UNIVERSIDAD DE VALENCIA Y JESÚS TEJADA GIMÉNEZ. VISIBILIDAD DE ESTA REVISTA: SCOPUS, EMERGING SOURCES CITATION INDEX (CLARIVATE), EBSCO, CINDOC (CSIC), CITEFACTOR, COPAC, DIALNET, DICE (CSIC), DOAJ, E-REVISTAS (CSIC), EBSCO PREMIER, ERIH+, GALE CENGAGE LEARNING, IN-RECS, IRESIE, LATINDEX, MIAR, OCLC WORLDCAT, RESH, REDIB, RILM CORE JOURNALS, SUDOC, ULRICHS, ESTA REVISTA ESTÁ PUBLICADA CON EL APOYO INSTITUCIONAL DE REDIRIS-CONSEJO SUPERIOR DE INVESTIGACIONES CIENTIFICAS Y ES DE ACCESO LIBRE. CREATIVE COMMONS LICENSE 4.O BY 
Capítulo 10. Pasión, perseverancia y... ¡adelante! Un capítulo final que ilustra a la perfección la importancia del esfuerzo constante, el deseo de conseguir la meta a pesar de los impedimentos y la pasión y la perseverancia que una persona puede llegar a alcanzar en su trabajo musical diario.

El libro concluye con un apartado dedicado a referencias bibliográficas y un exhaustivo índice onomástico que puede resultar de gran utilidad.

En definitiva, esta bien documentada monografía destaca por su carácter eminentemente práctico, donde se expone un tema de escaso tratamiento por parte de los expertos que es esencial en el largo y arduo aprendizaje de la interpretación musical. Todo músico debe enfrentarse a este proceso en soledad, no sólo durante y al final de sus estudios, sino también el resto de la vida; de ahí el valor del libro de la Dra. Tripiana.

\section{Referencias}

Barry, N.H. y Hallam, S. (2002). Practice. In R. Parncutt y G.E. McPherson (Eds.), The Science and Psychology of Music Performance: Creative Strategies for Teaching and Learning (pp.151-165). Oxford: Oxford University Press.

Gabrielsson, A. (2003). Music performance research at the millennium. Psychology of music, $31(3), 221-272$.

Jørgensen, H. (2004). Strategies for individual practice. In A. Williamon (Ed.), Musical excellence. Strategies and techniques to enhance performance (pp.85-103). London: Oxford University Press.

Santos, R.A.T. y Hentschke, L. (2009). A perspectiva pragmática nas pesquisas sobre prática instrumental: condições e implicações procedimentais. PER MUSI - Revista Acadêmica de Música, 19, 72-82. 